Commun. math. Phys. 5, 330-336 (1967)

\title{
Spontaneous Breakdown of Symmetries and Zero-Mass States*
}

\author{
H. EzAwA** \\ II. Institut für Theoretische Physik, Universität Hamburg, \\ Hamburg, Germany \\ and J. A. SwIECA*** \\ Department of Physics, University of Illinois, \\ Urbana, Illinois, U.S.A. \\ Received April 15, 1967
}

\begin{abstract}
In a relativistic field theory Goldstone's theorem is proved without any assumption about the existence of covariant fields and for arbitrary expectation values.
\end{abstract}

\section{Introduction}

In a recent paper [1] the problem of spontaneous breakdown of symmetries and the connection between algebraic automorphism and their unitary implementation in a Hilbert space was investigated.

In the framework of an algebraic field theory of the type formulated by HAAG and KASTLER [2], it was shown that automorphism connected with locally conserved currents are always unitarily implementable in a representation in which the energy-momentum spectrum has a gap between the vacuum and a lowest mass hyperboloid. Therefore a spontaneous breakdown of the symmetry which is associated with the conservation law can occur only if the energy-momentum spectrum has no such gap.

In the present note under the same assumptions as in [1] a stronger result is obtained: The automorphisms are unitarily implementable unless the mass spectrum has a $\delta\left(x^{2}\right)$-singularity at the light cone. This is closer to what is usually understood by Goldstone's theorem [3] than the result of [1].

* This work was supported in part by the National Science Foundation, U.S.A.

** Address after April 1, 1967: Department of Physics, Gakushuin University, Mejiro, Toshima-ku, Tokyo, Japan.

*** Present address: Department of Physics, University of São Paulo, São Paulo, Brazil. 
In our proof we shall employ a technique similar to the one originally used in [4] with the difference that no use of covariant field operators will be made.

Earlier discussions of these matters and further references may be found in $[5,6,7,8]$.

\section{The Goldstone Theorem}

We take over the definitions, assumptions and notation of [1]. Apart from technicalities the input is:

(i) Existence of current densities $j_{\mu}(x)$ satisfying a continuity equation $\partial^{\mu} j_{\mu}(x)=0$.

The quantities

$$
j_{\mu}(f)=\int j_{\mu}(x) f(x) d^{4} x
$$

are operators in a Hilbert space if $f$ is a suitable decent weight function. In the present note we shall omit the time smearing to simplify the notation since it can be reinstated in all the formulas without changing any conclusion.

(ii) Existence of a continuous representation of the translation group in space-time by unitary operators $U(a)$ whose generators are the energy-momentum operators. The energy-momentum spectrum is supposed to be confined to $\overline{V^{(+)}}$(closure of forward light cone). There shall be exactly one vector in the Hilbert space which is invariant under $U(a)^{1}$. This vector $\Omega$ corresponds to the vacuum state.

(iii) Covariance of $j_{\mu}(x)$ with respect to $U(a)$ :

$$
U(a) j_{\mu}(x) U^{-1}(a)=j_{\mu}(x+a) .
$$

Nothing is assumed about the transformation of the $j_{\mu}(x)$ under the homogenous Lorentz group. In particular they need not be a vector field.

(iv) Locality

$$
\left[j_{\mu}(x), j_{v}(y)\right]=0 \quad \text { if } x-y \text { is space-like . }
$$

We want to prove now the following:

Theorem

$$
\lim _{R \rightarrow \infty}\left\langle\Omega,\left[j_{0}\left(f_{R}, t\right), A\right] \Omega\right\rangle=0
$$

for all operators $A \in \mathfrak{A}$ unless there is particle of zero mass in the theory which is connected to the vacuum state by $j_{0}(x)$.

Here

$$
j_{0}\left(f_{R}, t\right)=\int j_{0}(\mathbf{x}, t) f_{R}(\mathbf{x}) d^{3} x
$$

1 The uniqueness of $\Omega$ is a matter of choice, not an assumption about the physical situation. It means that we choose to consider an irreducible representation of the algebra $\mathfrak{A}[1]$. With this choice the condition for a "spontaneous breakdown" is that the expectation values $\langle\Omega, A \Omega\rangle$ are not invariant under the automorphism. 
and $f_{R}$ is a function which vanishes for $|\mathbf{x}|>2 R$ and has the constant value $\mathbf{1}$ for $|\mathbf{x}|<R$. $\mathfrak{A}$ is the algebra of all local operators. The proviso in the theorem means that (1) holds unless the expression

$$
\int e^{-i p x}\left\langle\Omega,\left[j_{0}(x), A\right] \Omega\right\rangle d^{4} x
$$

contains a $\delta\left(p^{2}\right)$-singularity for some $A$. It is well-known that the presence of such a singularity implies the existence of a massless particle with states $|a\rangle$ such that

$$
\left\langle a\left|j_{0}(x)\right| \Omega\right\rangle \neq 0 .
$$

On the other hand, if (1) holds then the main theorem of Ref. [1] tells us that the expectation values $\langle\Omega, A \Omega\rangle$ are invariant under the automorphism, i.e., that the symmetry is not spontaneously broken.

Proof of Theorem. As a consequence of local commutativity and the fact that the energy-momentum spectrum is contained in the positive light-cone one can write a Jost-Lehmann-Dyson representation [9] for the commutator

$$
\begin{aligned}
\left\langle\Omega,\left[j_{0}(\mathbf{x}, t), A\right] \Omega\right\rangle= & \int_{0}^{\infty} d x^{2} \int d^{3} y \Delta\left(\mathbf{x}-\mathbf{y}, t ; x^{2}\right) \varrho_{1}\left(\varkappa^{2}, \mathbf{y}\right)+ \\
& +\int_{0}^{\infty} d x^{2} \int d^{3} y \frac{\partial}{\partial t} \Delta\left(\mathbf{x}-\mathbf{y}, t ; x^{2}\right) \varrho_{2}\left(\varkappa^{2}, \mathbf{y}\right) .
\end{aligned}
$$

In (3), $\varrho_{1}\left(x^{2}, y\right)$ and $\varrho_{2}\left(x^{2}, y\right)$ have compact support $D$ in $\mathbf{y}$ independent of $\varkappa^{2}$ due to the commutativity of $A$ with $j_{0}(\mathbf{x}, t)$ for sufficiently large space-like separations.

We can write $(i=1,2)$

$$
\varrho_{i}\left(\varkappa^{2}, \mathbf{y}\right)=\bar{\varrho}_{i}\left(\varkappa^{2}\right) \delta^{3}(\mathbf{y})+\boldsymbol{\nabla} \cdot \boldsymbol{\sigma}_{i}\left(\varkappa^{2}, \mathbf{y}\right),
$$

where $\boldsymbol{\sigma}_{i}\left(\varkappa^{2}, \mathbf{y}\right)$ also has compact support in the variable $\mathbf{y}$.

To verify this we note that

$$
\sigma_{i}^{(1)}\left(\varkappa^{2}, \mathbf{y}\right)=\int_{-\infty}^{y_{1}}\left\{\varrho_{i}\left(\varkappa^{2}, y_{1}^{\prime}, y_{3}\right)-\delta\left(y_{1}^{\prime}\right) \int_{-\infty}^{\infty} \varrho_{i}\left(\varkappa^{2}, y_{1}^{\prime \prime}, y_{2}, y_{3}\right) d y_{1}^{\prime \prime}\right\} d y_{1}^{\prime}
$$

has compact support and that

$$
\varrho_{i}\left(x^{2}, \mathbf{y}\right)=\delta\left(y_{1}\right) \int_{-\infty}^{\infty} \varrho_{i}\left(x^{2}, y_{1}^{\prime}, y_{2}, y_{3}\right) d y_{1}^{\prime}+\frac{\partial \sigma_{i}^{(1)}}{\partial y_{1}} .
$$

Repeating this procedure for the coefficient of $\delta\left(y_{1}\right)$ with respect to the coordinates $y_{2}, y_{3}$ one arrives at (4).

If we insert (4) and (3) into the left-hand side of (1) the contribution of $\boldsymbol{\nabla} \cdot \boldsymbol{\sigma}$ drops out for sufficiently large $R$ due to the fact that $\Delta\left(x ; x^{2}\right)$ vanishes outside the light cone. Hence we have for sufficiently large 
$R\left(R>R_{0}(t)\right)$

$$
\begin{aligned}
& \left\langle\Omega,\left[j_{0}\left(f_{R}, t\right), A\right] \Omega\right\rangle \\
& \quad=\int d x^{2} \int d^{3} x f_{R}(\mathrm{x})\left\{\bar{\varrho}_{1}\left(\varkappa^{2}\right) \Delta\left(\mathrm{x}, t ; x^{2}\right)+\bar{\varrho}_{2}\left(x^{2}\right) \frac{\partial}{\partial t} \Lambda\left(\mathrm{x}, t ; x^{2}\right)\right\} .
\end{aligned}
$$

On the other hand we know that for $R>R_{0}(t)$,

for any finite $t$.

$$
\frac{\partial}{\partial t}\left\langle\Omega,\left[j_{0}\left(f_{R}, t\right), A\right] \Omega\right\rangle=0
$$

This follows directly from the continuity equation and locality (see lemma II in [1]). Inserting (7) we get the following conditions for the weight functions $\bar{\varrho}_{i}\left(\varkappa^{2}\right)$ which have to be satisfied identically in $t$ (as relations for tempered distributions):

This implies

$$
\begin{aligned}
& \int_{0}^{\infty} \bar{\varrho}_{1}\left(x^{2}\right) \cos x t d x^{2}=0, \\
& \int_{0}^{\infty} \bar{\varrho}_{2}\left(\varkappa^{2}\right) \varkappa \sin \varkappa t d x^{2}=0 .
\end{aligned}
$$

$$
\begin{aligned}
& \bar{\varrho}_{1}\left(\varkappa^{2}\right)=0, \\
& \bar{\varrho}_{2}\left(\varkappa^{2}\right)=\lambda \delta\left(\varkappa^{2}\right) .
\end{aligned}
$$

Therefore, with (7) and (10) we get

unless $\lambda \neq 0$ in

$$
\lim _{R \rightarrow \infty}\left\langle\Omega,\left[j_{0}\left(f_{R}, t\right), A\right] \Omega\right\rangle=0
$$

$$
\int \varrho_{2}\left(x^{2}, \mathbf{y}\right) d^{3} y=\bar{\varrho}_{2}\left(x^{2}\right)=\lambda \delta\left(x^{2}\right) .
$$

Summarizing we know that $\varrho_{2}\left(x^{2}, \mathbf{y}\right)$ has a compact support in $\mathbf{y}$ and its integral gives rise to a $\delta$-singularity in $\varkappa^{2}$ when the symmetry is broken.

In order to relate the above conclusion to the singularity of (2), we have to look into a measure theoretical property of $\varrho_{2}\left(x^{2}, y\right)$. Consider

$$
J_{+}[\varphi]=\int d^{4} x \varphi(x)\left\langle\Omega, j_{0}(x) A \Omega\right\rangle
$$

with a test function $\varphi(x)$ whose Fourier transform $\tilde{\varphi}(p)$ has a compact support in $R^{4}$.

Take a function $\tilde{\psi}(p)$ from $\mathfrak{S}$ such that

$$
\tilde{\psi}(p)=1 \text { when } p \in \operatorname{supp} \tilde{\varphi}(p),
$$

and let its Fourier transform be $\psi(-z)$, then

$$
\begin{aligned}
J_{+}[\varphi] & =\int d^{4} y d^{4} z \varphi(y)\left\langle\Omega, j_{0}(y-z) A \Omega\right\rangle \psi(-z) \\
& =\int d^{4} y d^{4} z \varphi(y) \psi(z)\left\langle\Omega, j_{0}(z) U^{-1}(y) A \Omega\right\rangle,
\end{aligned}
$$

where we have used the assumption (ii) to write [10]

$$
U^{-1}(y)=\int e^{-i p y} d E(p)
$$


with a projection-valued measure $E$ on the momentum space. Then

where

$$
J_{+}[\varphi]=\int \tilde{\varphi}(p) d \mu_{+}(p),
$$

$$
d \mu_{+}(p)=\langle\Psi, d E(p) \Phi\rangle
$$

is a $c$-number measure as defined with the normalizable vectors,

$$
\Phi=A \Omega \quad \text { and } \quad \Psi=\int d^{4} z \psi^{*}(z) j_{0}(z) \Omega .
$$

Repeating the same process for the product $A j_{0}(x)$, we can establish that the functional

can be written as

$$
I[\varphi]=\int d^{4} x \varphi(x)\left\langle\Omega,\left[j_{0}(x), A\right] \Omega\right\rangle
$$

where $\mu$ is a measure.

$$
I[\varphi]=\int \tilde{\varphi}(p) d \mu(p)
$$

The fact that $I[\varphi]$ can be written in terms of a measure is essential in the following argument. The weight in the Jost-Lehmann-Dyson representation (3) can be identified as

$$
d \mu(p)=\left[\varrho_{1}\left(p^{2}, \mathbf{p}\right)+i p_{0} \varrho_{2}\left(p^{2}, \mathbf{p}\right)\right] \varepsilon(p) \psi(p) d^{4} p .
$$

In order to look more closely at the behaviour of $\mu$ in the neighborhood of the zero-mass cone $p^{2}=0$, we take a set of test functions with a parameter $\gamma>0$,

$$
\varphi_{\gamma}(p)=\tilde{h}(\mathbf{p}) \Lambda\left(p^{2} ; \gamma\right),
$$

where both $\tilde{h}$ and $\Lambda$ are continuous and

$$
\begin{aligned}
\Lambda\left(p^{2} ; \gamma\right) & =0 \text { for }\left|p^{2}\right|>\gamma, \\
\Lambda(0 ; \gamma) & =1 .
\end{aligned}
$$

Since these test functions are symmetric with respect to $p_{0}$, the weight $\varrho_{1}$ gives no contribution to (15). Changing the variable of integration we get

$$
I\left[\varphi_{\gamma}\right]=i \int d p^{2} d \mathbf{p} \tilde{h}(\mathbf{p}) \Lambda\left(p^{2} ; \gamma\right) \varrho_{2}\left(p^{2} ; \mathbf{p}\right) .
$$

In the sharp-mass limit $\gamma \rightarrow 0$, we have

$$
\lim _{\gamma \rightarrow 0} I\left[\varphi_{\gamma}\right]=\int d \mathbf{p} \tilde{h}(\mathbf{p}) \tilde{\alpha}(\mathbf{p}),
$$

where use has been made of $(4)$ and (10), and

$$
\begin{aligned}
\tilde{\alpha}(\mathbf{p}) & =i \lambda+\mathbf{p} \cdot \tilde{\boldsymbol{\beta}}(\mathbf{p}), \\
\tilde{\boldsymbol{\beta}}(\mathbf{p}) & =\lim _{\gamma \rightarrow 0} \int d p^{2} \Lambda\left(p^{2} ; \gamma\right) \boldsymbol{\sigma}_{2}\left(p^{2}, \mathbf{p}\right) .
\end{aligned}
$$

In fact, the orders of the integrations and the limit can be changed because $\tilde{h} \Lambda$ is bounded and $\varrho_{2}$ is defining a measure. Now, recall that the support in $\mathbf{y}$ of $\sigma_{2}\left(p^{2}, \mathbf{y}\right)$ is bounded uniformly with respect to $p^{2}$. Then, 
the support of the Fourier transform $\boldsymbol{\beta}(\mathbf{y})$ of $\tilde{\boldsymbol{\beta}}(\mathbf{p})$ is compact, which in turn implies that $\tilde{\boldsymbol{\beta}}(\mathbf{p})$ is entire analytic in $\mathbf{p}$. Hence, $\mathbf{p} \cdot \tilde{\boldsymbol{\beta}}(\mathbf{p})$ in $(21)$ cannot be a constant $\neq 0$, so that $\tilde{\alpha}(\mathbf{p})$ does not vanish identically, if $\lambda \neq 0$, in any neighborhood of every point $\mathbf{p}$ in momentum space. Let $S$ denote the open set of the point $\mathbf{p}$ for which $\tilde{\alpha}(\mathbf{p}) \neq 0$. Take a function $\tilde{h}(\mathbf{p})=f(\mathbf{p} ; \dot{\mathbf{p}}) \geqq 0$ whose support is contained in a sufficiently small sphere centered at $\dot{\mathbf{p}} \in S$, then we see from (20) that

$$
\lim _{\gamma \rightarrow 0} I\left[\varphi_{\gamma}\right] \neq 0 \text { if } \lambda \neq 0 .
$$

The proof can now be concluded by appealing to a general property of measure. Any measure $\mu$ can be decomposed into three parts $\mu=\mu_{1}+$ $+\mu_{2}+\mu_{3}$, where $\mu_{1}$ is $L$-absolutely continuous, $\mu_{2}$ is continuous but $L$-singular and $\mu_{3}$ is $L$-purely discontinuous (Lebesgue decomposition) [11]. Restricting ourselves to the test function (17) that is symmetric in $p_{0}$, we write $\mu(p)$ as $\mu\left(p^{2}, \mathbf{p}\right)$, to which the Lebesgue decomposition is applied. In the sharp-mass limit $\gamma \rightarrow 0$, we know from the definition of the $L$-continuity that $\lim _{\gamma \rightarrow 0} I\left[\varphi_{\gamma}\right]$ cannot be non-vanishing unless our measure $\mu$ contains a purely discontinuous part, which is $\delta\left(p^{2}\right)$. Thus, we conclude finally that, when the symmetry is broken, the measure $\mu\left(p^{2}, \mathbf{p}\right)$ has the desired $\delta\left(p^{2}\right)$-singularity at least for such $\mathbf{p}$ that makes $\tilde{\alpha}(\mathbf{p}) \neq 0$. This concludes the proof of the theorem.

To understand the essential ingredients of our argument it may be helpful to compare it with the following naive proof of Goldstone's theorem.

Consider the distribution

$$
L\left(\mathbf{p}, p_{0}\right)=\int\left\langle\Omega,\left[j_{0}(\mathbf{x}, t), A\right] \Omega\right\rangle e^{-i \mathbf{p} \mathbf{x}+i p_{0} t} d^{3} x d t .
$$

Using the continuity equation and locality one concludes as in eq. (8)

and hence

$$
\lim _{\mathbf{p} \rightarrow 0} p_{0} L\left(\mathbf{p}, p_{0}\right)=0
$$

$$
L\left(\mathbf{0}, p_{0}\right)=\lambda \delta\left(p_{0}\right) .
$$

This will imply the existence of a discrete excitation whose energy goes to zero with the momentum only if one knows that $L\left(\mathbf{p}, p_{0}\right)$ can be written as $g\left(\mathbf{p} ; p_{0}-E(\mathbf{p})\right)$ where $g$ is smooth in its first variable.

This is always the case for relativistic field theories as a consequence of local commutativity because $\varrho_{1,2}\left(p^{2}, \mathbf{p}\right)$ is the Fourier transform of a function with compact support and therefore analytic in $\mathbf{p}$.

Acknowledgements. The authors are most indebted to Professor R. HAA for his constant interest and advices and to Dr. O. SteinmanN for his help and valuable comments. The financial support of the National Science Foundation, U.S.A. is gratefully acknowledged. 


\section{References}

1. Kastler, D., D. Robinson, and A. Swieca: Commun. Math. Phys. 2, 108 (1966).

2. HAAG, R., and D. Kastler: J. Math. Phys. 5, 848 (1964).

3. Goldstone, J.: Nuovo Cimento 19, 154 (1961).

4. -, A. Salam, and S. Weinberg: Phys. Rev. 127, 965 (1962).

5. Guralnik, G. S., T. Kibble, and C. R. Hagen: Phys. Rev. Letters 13, 585 (1964).

6. Lange, R. V.: Phys. Rev. Letters 14, 3 (1965).

7. Streater, R. F.: Trieste Lectures, May 1965 (published by I.C.E.A., Trieste).

8. Kibble, T.: Oxford Conference Report 1965.

9. Jost, R., and H. Lehmann: Nuovo Cimento 5, 1598 (1957); Dyson, F. J.: Phys. Rev. 110, 1460 (1958); Araki, H., K. Hepp, and D. Ruelle: Helv. Phys. Acta 35, 164 (1962).

10. Streater, R. F., and A. S. Wightman: PCT, spin and statistics and all that, p. 92. New York: Benjamin Inc. 1964; Jost, R.: The general theory of quantized fields, p. 53. Am. Math. Soc., Providence: 1965.

11. Iтo, S.: Introduction to Lebesgue integral, p. 134. Tokyo: Syôkabô 1963 (in Japanese); " $L$ " means "with respect to the Lebesgue measure". 